\title{
A method for determining by direct renormalization the properties of long polymers in solutions
}

\author{
J. des Cloizeaux \\ Service de Physique Théorique, CEN Saclay, BP no 2, 91190 Gif-sur-Yvette, France \\ (Reçu le 22 janvier 1980, accepté le 18 février 1980)
}

\begin{abstract}
Résumé. - On montre que les propriétés de longs polymères peuvent être déterminées par renormalisation directe de quantités fondamentales telles que distances bout à bout de polymères et coefficients du viriel. Comme exemple, on calcule le second coefficient du viriel d'une solution de polymères monodisperses et les indices critiques $\gamma, v$ et $\omega$ au premier ordre en $\varepsilon=4-d(d=$ dimension de l'espace). La méthode est tout à fait générale et s'applique aussi à des polymères polydisperses.
\end{abstract}

\begin{abstract}
It is shown that the properties of long polymers in a solution can be determined by direct renormalization of fundamental quantities such as end to end distances of polymers and virial coefficients. As an example, the second virial coefficient of a monodisperse polymer solution and the critical indices $\gamma, v$ and $\omega$, are calulated to first order in $\varepsilon=4-d(d=$ dimension of space). The method is quite general and applies also to polydisperse polymers.
\end{abstract}

1. Introduction. - A very long polymer, in a solution, is a critical object. This means that its properties depend only on one parameter, the end to end distance. In the same way, an infinitely long chain is a statistically unique object.

This fact has far reaching consequences, because it implies the existence of critical indices and scaling laws; however, it has been only gradually recognized.

The process started in 1934. At that time, W. Kuhn realized that excluded volume produces anomalous effects. In a remarkable article [1], he introduced the index which is now called $v$ and he gave for it, the value 0.61 . In 1949, P. Flory produced simple arguments leading to a slightly more accurate value $3 / 5$. Unfortunately, the success of this approach (in spite of imperfections [3]) and the lack of precise experiments prevented for many years all fundamental progress.

The next break-through occurred in 1972. Then, P. G. de Gennes discovered [4] the analogy which exists between an isolated polymer and a magnetic system, in a vanishing external field, when the number of components of the magnetization goes to zero.

Thus, the renormalization techniques developed in field theory and used by K. Wilson and his followers $[5,6]$ could be directly applied to polymer solutions. This magnetic analogy has been extended by the author [7] who showed that a polymer solution corresponds to a magnetic system in an external magnetic field. However, the analogy is rather artificial and, as was first pointed out by P. G. de Gennes [8], with this method, polydispersion effects cannot be handled in a satisfactory way.

Thus, the whole process would be much clearer if the renormalization concepts could be applied directly to the study of polymer solutions. The purpose of this letter is just to show how this aim can be reached. Fundamentally, we shall follow the analytic renormalization methods used by Brézin, le Guillou and Zinn-Justin [9] but without referring to any magnetic system.

Incidentally, we may note that several authors have been concerned with this problem. The same trend towards direct renormalization appears in very recent articles by L. Schäfer and T. Witten [10] and by D. J. Elderfield [11].

We may note that the method which is presented here does not use the idea of renormalization along the chain, which has been described in 1977 by P. G. de Gennes [12] and exploited, in 1978 by M. Gabay and T. Garel [13] and, in 1979 by Y. Oono [14]. This other approach does not seem very convenient and in particular the block renormalization technique always introduces ambiguities which are not easily solved.

On the contrary, the method presented here relies on techniques which have been used with great 
success in field theory, and for this reason the new method appears as really powerful and flexible. In particular it might be useful to study the static or dynamical properties of various kinds of monodisperse or polydisperse polymers in solutions.

2. Notation. - Polymers in good solvents are represented by Brownian chains with excluded volume. The weight associated with a configuration containing many chains is obtained by direct generalization of the weight associated with one chain. This weight can be defined as follows :

$$
\begin{aligned}
P=\exp \{- & \frac{1}{2} \int_{0}^{S}\left[\frac{\mathrm{d} \mathbf{r}(s)}{\mathrm{d} s}\right]^{2} \mathrm{~d} s- \\
& \left.\quad-\frac{b}{2} \int_{0}^{S} \mathrm{~d} s^{\prime} \int_{0}^{S} \mathrm{~d} s^{\prime \prime} \delta\left[\mathrm{I}\left(s^{\prime}\right)-\mathrm{I}\left(s^{\prime \prime}\right)\right]\right\} .
\end{aligned}
$$

In the absence of interaction $(b=0)$, we have

$$
\begin{aligned}
R^{2} \equiv\left\langle[\mathrm{r}(S)-\mathrm{r}(0)]^{2}\right\rangle & =d S \\
(d & =\text { dimension of space }) .
\end{aligned}
$$

Thus, the size of the Brownian chain is defined by the surface $S$ and this fact will not surprise the reader if he remembers that the Hausdorff dimension of a Brownian chain is 2 .

The parameters $S$ and $b$ are given in terms of the number of links $N$, the characteristic length $l$ and the excluded volume $u$ (a volume for $d=3$ but a surface for $d=2$ ) by

$$
S=N l^{2} \quad b=u l^{-4} .
$$

The number of configurations of an assembly of $\mathbf{N}$ chains submitted to given constraints can be represented by the symbol $3(\ldots)$. To calculate a number $\tilde{\jmath}(\ldots)$, a cut-off $s_{0}=n_{0} l^{2}$ has to be introduced; then, $\tilde{J}(. .$.$) can be written as follows [15] (ultraviolet regu-$ larization) :

$\tilde{\jmath}(\ldots)=\exp \left[s_{0}^{-1} C\left(b s_{0}^{\varepsilon / 2}\right) \sum_{1}^{N} S_{i}\right] \cdot \xi(\ldots) \quad(\varepsilon=4-d)$.

The entire dependence on $s_{0}$ (or $n_{0}$ ) is given by $C\left(b s_{0}^{\varepsilon / 2}\right)$ and $\cdot 3(\ldots)$ does not depend on $s_{0}\left(n_{0} / N \ll 1\right)$. The quantity $\cdot 3(. .$.$) can be expanded with respect to b$, in a convergent way, and represented by diagrams.

It will be particularly convenient to define the partition function $\kappa(. .$.

$$
\oiiint(\ldots)=l^{d(\mathbf{N}-1)} \cdot \zeta_{\mathrm{c}}(\ldots)
$$

which are proportional to the connected part $\bullet 3_{\mathrm{c}}(\ldots)$ of $\cdot z(\ldots)$.

In fact, when the polymers are monodisperse, $\varkappa(\ldots)$ depends only on $S$ and $b$ and the only parameter in the theory [16] is the well known quantity

$$
z=b S^{2-d / 2}(2 \pi)^{-d / 2}=B S^{\varepsilon / 2} \quad(\varepsilon=4-d) .
$$

The properties of monodisperse solution depend also on the number $\mathbf{C}$ of polymers per unit volume and the quantity which represents the concentration in monomers is the inverse of a length $\mathbf{C S}=L^{-1}$.

3. Perturbation expansion. - The partition function of $\mathbf{N}$ chains defined by the surfaces $S_{1}, \ldots, S_{\mathbf{N}}$ is $\pi\left(S_{1}, \ldots, S_{\mathbf{N}}\right)$ and the expansion of this quantity can be represented by single diagrams (see Fig. 1) :

1) A diagram consists of $\mathbf{N}$ polymer lines connected by interaction lines.

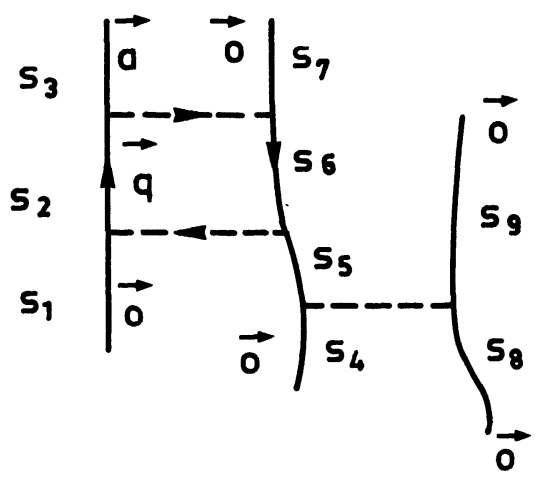

Fig. 1. - A diagram contributing to $\pi\left(S_{1}, S_{2}, S_{3}\right)$

$$
\begin{aligned}
& S_{1}=s_{1}+s_{2}+s_{3} \\
& S_{2}=s_{4}+s_{5}+s_{6}+s_{7} \\
& S_{3}=s_{8}+s_{9} .
\end{aligned}
$$

2) Each polymer segment and each interaction line carries a wave vector and the wave vector flow is conserved at each interaction point.

3) The sum of the surfaces of the polymer segments belonging to the polymer line of index $j$ is $S_{j}$.

4) $\mathrm{A}$ factor $\mathrm{e}^{-k^{2} s / 2}$ is associated with each polymer segment of surface $s$ and wave vector $\mathbf{k}$.

5) A factor $-b(2 \pi)^{-d}$ is associated with each interaction line.

6) A global factor $(2 \pi)^{d(\mathbf{N}-1)}$ is associated with the diagram itself.

7) Symmetry factors are introduced as usual. (In the following, the polymers will be assumed to be disymmetric and discernable.)

8) The diagram is calculated by integrating the product over all the independent wave vectors and surfaces. The integrals over surfaces are regularized; for any $n$ (for instance $n=-d / 2$ ), we write

$$
\int^{s} \mathrm{~d} s s^{n}=S^{n+1} /(n+1) .
$$

The other partition functions $\varkappa(\ldots)$ are calculated in a similar way. 
4. Renormalization factors and critical indices. Let us consider an isolated chain. If $b=0$, the chain is Brownian, but if $S^{\varepsilon / 2} b$ becomes large, we approach another asymptotic limit. In this limit, the excluded volume effects are dominant : the chain is Kuhnian. Then, we have

$$
R^{2} \equiv\left\langle[\mathbf{r}(S)-\mathbf{r}(0)]^{2}\right\rangle=d^{\mathrm{e}} S
$$

where ${ }^{\mathrm{e}} S$ is the new scaling surface.

In the asymptotic limit, the bare partition functions $\varkappa(. .$.$) do not obey simple scaling laws. However, they$ are proportional to other functions for which these scaling laws apply. These new functions are the renormalized partition functions $\varkappa_{R}(\ldots)$.

More precisely, if the diagrams contributing to $\varkappa(. .$.$) , contain external vertices (also called insertions;$ see Fig. 2), the vertices have to be renormalized (as in field theory where vertex renormalization is the rule).
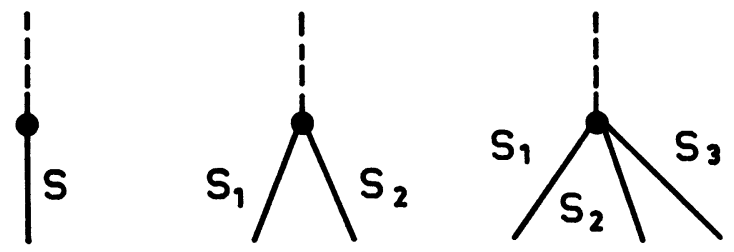

Fig. 2. - Vertices or insertions. A wave vector is inserted in the diagram at the vertex. If the wave vector is zero, the dashed line can be omitted as in figure 1 .

The free end of a polymer of surface $S$ is a 1-vertex and the corresponding renormalization factor will denoted by $X_{1}(S)$. In the same way, a point from which start $\mathbf{N}$ polymers of surfaces $S_{1}, \ldots, S_{\mathrm{N}}$ is an $\mathbf{N}$-vertex (or star) and the corresponding renormalization factor can be denoted by $X_{\mathrm{N}}\left(S_{1}, \ldots, S_{\mathrm{N}}\right)$. A simplification occurs when $S_{1}, \ldots, S_{\mathrm{N}}$ are of the same order of magnitude. In this case, simpler renormalization factors $X_{\mathrm{N}}(S)$ can be defined (for instance) by writing

$$
\begin{aligned}
X_{\mathbf{N}}(S)= & S^{-\mathbf{N}+1} \int_{S_{i}>0} \mathrm{~d} S_{1} \ldots \mathrm{d} S_{\mathbf{N}} \\
& \delta\left(S_{1}+\cdots+S_{\mathbf{N}}-S\right) X_{\mathbf{N}}\left(S_{1}, \ldots, S_{\mathbf{N}}\right) .
\end{aligned}
$$

The renormalization factors are numbers and are determined by renormalization conditions.

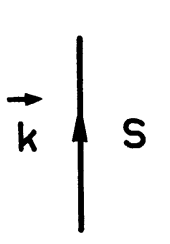

(a)

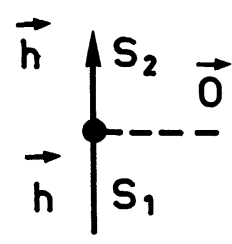

(b)
Fig. 3. - a) Diagram without insertion. Contribution : $\left.\exp \left(-S k^{2} / 2\right) ; b\right)$ The same diagram with a 2-vertex insertion. Contribution : $\exp \left[-\left(S_{1}+S_{2}\right) h^{2} / 2\right]$. As $S=S_{1}+S_{2}$, these contributions are equal.
We note that inserting a 2-vertex of wave vector zero on a diagram does not change anything in the calculation of the diagram (see a trivial example on Fig. 3). Thus, we have

$$
X_{2}\left(S_{1}, S_{2}\right)=1 \quad X_{2}(S)=1
$$

Such a situation does not occur in current field theory. It indicates that another renormalization factor (related to the index $v$ ) must be introduced. We define this fundamental renormalization factor $X_{0}(S)$ (swelling factor) by setting [17]

$$
X_{0}(S)={ }^{\mathrm{e}} S / S
$$

For large values of $S^{\varepsilon / 2} b$, the renormalization factors behave as follows

$$
X_{\mathrm{N}}(S) \propto\left({ }^{\mathrm{e}} S b^{2 / \varepsilon}\right)^{\kappa_{\mathrm{N}}}
$$

The coefficients $\kappa_{\mathbf{N}}$ are critical indices which depend only on $d$.

5. Renormalization conditions. - As we shall see, the current indices $\gamma$ and $v$ are directly related to $\kappa_{0}$ and $\kappa_{1}$ and to determine them, we have to define $X_{0}(S)$ and $X_{1}(S)$ more precisely.

For this purpose, three partition functions will be useful.

a) $\varkappa(S)$ and $\varkappa(S, S)$

b) the Fourier transform $\nVdash(S ; \mathbf{k},-\mathbf{k})$ of the partition function $K\left(S ; r_{1}, r_{2}\right)$ of an isolated chain of surface $S$, of origin $r_{1}$ and of extremity $r_{2}$ :

$$
\pi(S ; \mathbf{k},-\mathbf{k})=\mathcal{H}(S)\left\langle\mathrm{e}^{i \mathbf{k} .\left(\mathbf{r}_{1}-\mathbf{r}_{2}\right)}\right\rangle
$$

This equation leads to

$$
{ }^{\mathrm{e}} S=-\frac{2}{\kappa(S)}\left[\frac{\hat{c}}{\hat{c} k^{2}} \mathfrak{\kappa}(S ; \mathbf{k},-\mathbf{k})\right]_{k^{2}=0}
$$

The corresponding renormalized quantities are given by

$$
\begin{gathered}
\varkappa(S ; \mathbf{k},-\mathbf{k})=\left(X_{1}\right)^{2} \varkappa_{\mathrm{R}}(S ; \mathbf{k},-\mathbf{k}) \\
\varkappa(S, S)=\left(X_{1}\right)^{4} \varkappa_{\mathrm{R}}(S ; S) .
\end{gathered}
$$

The renormalization condition for $X_{1}$ is

$$
\varkappa_{\mathrm{R}}(S)=1 ; \quad X_{1}^{2}=\varkappa(S)
$$

and $X_{0}$ is defined by eq. (10).

On the other hand, the effective interaction is given by $\mathscr{K}_{\mathrm{R}}(S, S)$ which is proportional to the second virial coefficient and can be written

$$
\varkappa_{\mathrm{R}}(S, S)=-\left({ }^{\mathrm{e}} S\right)^{d / 2}(2 \pi)^{d / 2} g
$$

where $g$ is a dimensionless constant.

Now, it is possible to express $X_{0}$ and $X_{1}$ in terms of the variables ' $S$ and $g$ which, in the asymptotic limit, have a deep physical meaning. 
6. Identification of the critical indices. - Let us show that $\kappa_{0}$ and $\kappa_{1}$ are related to the current indices $v$ and $\gamma$.

In the asymptotic limit $(N \rightarrow \infty)$, we have by definition

$$
S \propto N \quad{ }^{\mathrm{e}} S \propto N^{2 v} \quad \varkappa(S) \propto N^{\eta-1} .
$$

Therefore, eqs. (10), (11) give

$$
\kappa_{0}=1-\frac{1}{2 v}
$$

and eqs. (16), (11) give

$$
\kappa_{1}=\frac{\gamma-1}{4 v} .
$$

7. Fixed point. Asymptotic value of the second virial coefficient. - Let us consider the function

$$
W(g)=\left.2{ }^{\mathrm{e}} S \frac{\partial g}{\partial^{\mathrm{e}} S}\right|_{b=\text { const. }} .
$$

The value $g^{*}$ of $g$ at the fixed point is defined by

$$
W\left(g^{*}\right)=0 .
$$

The critical index $\omega$ which determines the nature of the corrections to scaling [18], is given by

$$
\omega=-\left.\frac{\hat{c} W(g)}{\hat{c} g}\right|_{g=g^{*}}
$$

On the other hand, effective indices $\kappa_{\mathrm{N}}(g)$ can be defined by

$$
\kappa_{\mathbf{N}}(g)=\left.{ }^{\mathrm{e}} S \frac{\hat{c}}{\partial{ }^{\mathrm{e}} S} \ln X_{\mathbf{N}}\right|_{b=\text { const. }}
$$

and the critical indices are given by

$$
\kappa_{\mathrm{N}}=\kappa_{\mathrm{N}}\left(g^{*}\right) \text {. }
$$

Let us now consider the osmotic pressure $\Pi$ of a solution of monodisperse polymers. The first two terms of the expansion of $\Pi$ with respect to the number $\mathbf{C}$ of polymers per unit volume, are

$$
\Pi \beta=\mathbf{C}-\frac{1}{2} \mathbf{C}^{2} \frac{\pi(S, S)}{[\pi(S)]^{2}}=\mathbf{C}-\frac{1}{2} \mathbf{C}^{2} \frac{\kappa_{\mathrm{R}}(S, S)}{\left[\kappa_{\mathrm{R}}(S)\right]^{2}}
$$

where $\mathbf{C}$ is the number of polymers per unit volume. In the asymptotic limit, we get

$$
\Pi \beta=\mathbf{C}\left[1+\frac{1}{2}(2 \pi)^{d / 2} g^{*}\left(\mathbf{C}^{\mathrm{e}} S^{d / 2}\right)+\cdots\right]
$$

which is the first term of the expansion of

$$
\Pi \beta=\mathbf{C} \varphi\left(\mathbf{C}^{\mathrm{e}} S^{d / 2}\right)
$$

where $\varphi(x)$ is a universal function

$$
\left(\varphi(x) \propto x^{1 /(v d-1)} x \rightarrow \infty\right) \quad[3] .
$$

8. First order approximation. - In order to illustrate the method, we shall apply it near $d=4$, to first order with respect to $\varepsilon=4-d>0$. The one loop diagrams contributing to $\kappa(S ; \mathbf{k},-\mathbf{k})$ and $\varkappa(S, S)$ are given on figures 4 and 5 .

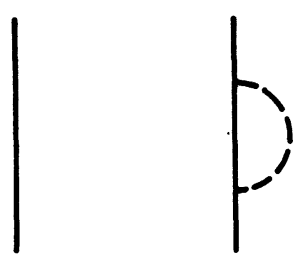

Fig. 4. - Diagrams contributing to $\varkappa(S ; k,-k)$.
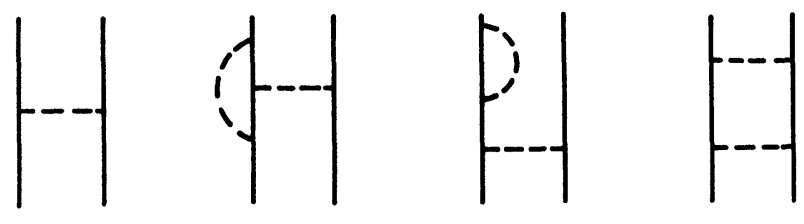

Fig. 5. - Diagrams contributing to $\varkappa(S, S)$.

Setting $B=b(2 \pi)^{-d / 2}$, we find (k small)

$\varkappa(S)=\varkappa(S ; 0,0)$

$\kappa(S ; \mathbf{k},-\mathbf{k}) \simeq 1+B S^{\varepsilon / 2}\left(\frac{2}{\varepsilon}\right)-$

$-k^{2}\left[\frac{S}{2}+B S^{1+\varepsilon / 2}\left(\frac{2}{\varepsilon}\right)\right]+\cdots$

$\varkappa(S, S) \simeq-B(2 \pi)^{d / 2} S^{2}\left[1+B S^{\varepsilon / 2}\left(\frac{0}{\varepsilon}\right)+\cdots\right]$

Eqs. (13), (10), (14), (16) and (29) give

$$
{ }^{\mathrm{e}} S \simeq S\left[1+B S^{\varepsilon / 2}\left(\frac{2}{\varepsilon}\right)\right]
$$

$X_{0}(S) \simeq 1+B S^{\varepsilon / 2}\left(\frac{2}{\varepsilon}\right) \simeq 1+B\left({ }^{\mathrm{e}} S\right)^{\varepsilon / 2}\left(\frac{2}{\varepsilon}\right)$

$X_{1}(S) \simeq 1+B S^{\varepsilon / 2}\left(\frac{1}{\varepsilon}\right) \simeq 1+B\left({ }^{\mathrm{e}} S\right)^{\varepsilon / 2}\left(\frac{1}{\varepsilon}\right)$

On the other hand, eqs. (15), (17) and (30) lead to

$$
\left(X_{1}\right)^{4} g\left({ }^{\mathrm{e}} S\right)^{2-\varepsilon / 2} \simeq B S^{2}
$$

which will the help of eqs. (31) and (32) give

$$
g \simeq B\left({ }^{\mathrm{e}} S\right)^{\varepsilon / 2}\left[1-B\left({ }^{\mathrm{e}} S\right)^{\varepsilon / 2}\left(\frac{8}{\varepsilon}\right)\right]
$$


From eq. (21), we obtain

$$
\begin{aligned}
W(g) & \simeq \varepsilon B\left({ }^{\mathrm{e}} S\right)^{\varepsilon / 2}\left[1-B\left({ }^{\mathrm{e}} S\right)^{\varepsilon / 2}\left(\frac{16}{\varepsilon}\right)\right] \\
& \simeq \varepsilon g\left(1-\frac{8 g}{\varepsilon}\right) .
\end{aligned}
$$

The equation $W\left(g^{*}\right)=0$, gives

$$
g^{*} \simeq \frac{\varepsilon}{8} \text {. }
$$

In this way, we find the asymptotic second virial coefficient to order $\varepsilon$ and a comparison with the results obtained by Witten and Schäfer [19] and by Elderfield [20] should be possible.

Let us now calculate $\kappa_{0}$ and $\kappa_{1}$. Eqs. (24), (31) and (32) give

$$
\begin{aligned}
& \kappa_{0}(g)=B\left({ }^{\mathrm{e}} S\right)^{\varepsilon / 2} \simeq g \\
& \kappa_{1}(g)=\frac{B}{2}\left({ }^{\mathrm{e}} S\right)^{\varepsilon / 2} \simeq \frac{g}{2}
\end{aligned}
$$

\section{References}

[1] KuHn, W., Kolloid Z. 68 (1934) 2.

[2] FloRy, P. J., Principles of Polymer Chemistry (Cornell University Press) 1971 , Ch. XIV, p. 596.

[3] Des Cloizeaux, J., J. Physique 31 (1970) 715 and 37 (1976) 431

[4] De Gennes, P. G., Phys. Lett. 38A (1972) 339.

[5] See Phase Transitions and Critical Phenomena, vol. 6, Edited by Domb, C. and Green, M. S. (Academic Press) 1976.

[6] See Field Theory, the Renormalization Group and Critical Phenomena by AмIт, D. J. (McGraw Hill) 1978.

[7] Des Cloizeaux, J., J. Physique 36 (1975) 281.

[8] De Gennes, P. G., Private Communication.

[9] Brézin, E., Le Guillou, J. C. and Zinn-Justin, J., See Ref. [5], p. 125 .

[10] SchäFer, L. and Witten, T., Preprint to be published in $J$. Physique.

[11] Elderfield, D. J., Preprint.

[12] De Gennes, P. G., Riv. Nuovo Cimento 7 (1977) 123.

[13] Gabay, M. and Garel, T., J. Physique Lett. 39 (1978) L-123.

[14] Oono, Y., J. Phys. Soc. Japan 47 (1979) 683. and for $g=g^{*}$, we obtain

$$
\kappa_{0} \simeq \frac{\varepsilon}{8} ; \quad \kappa_{1} \simeq \frac{\varepsilon}{16} .
$$

Finally, eqs. (19) and (20) lead to the well known results $[5]$

$$
v \simeq \frac{1}{2}\left(1+\frac{\varepsilon}{8}\right), \quad \gamma=1+\frac{\varepsilon}{8} .
$$

9. Conclusion. - The method of direct of renormalization which is described here can be interesting for two reasons;

a) From a theoretical point of view, it shows that the properties of long polymers in solutions can be studied directly without any reference to the zero component Lagrangian theory. In this way, the implications of the theory become more transparent.

b) From a practical point of view, this approach can be used for describing the behaviour of any system of polymers of given polydispersion.

The author intends to give more details concerning the method and the applications in future publications.

[15] Des Cloizeaux, J., Submitted to the J. Physique.

[16] Yamakawa, H., Modern Theory of Polymer Solutions (Harper and Row) 1971, p. 84.

[17] Eq. (10) of the present article is similar to the second eq. (2.3) of Des Cloizeaux, J. Phys. Rev. A 10 (1974) 1665.

[18] Our $\omega$ coincide with the index $\omega$ defined by Brézin, Le Guillou and Zinn-Justin (ref. [8]).

[19] Witten, T. A. and Schäfer, L., J. Phys. A-Math. Gen. 11 (1978) 1843. For $d=3, \psi=\frac{g}{8}\left(\frac{2 R^{2}}{3 R_{\mathrm{G}}^{2}}\right)^{3 / 2}$ and setting $R^{2} / R_{\mathrm{G}}^{2} \simeq 6$, we find $\psi=0.125$ whereas they find in first order $\psi=0.140$ (but changing the prefactors may change the results !).

[20] Elderfield, D. J., J. Phys. A-Math. Gen. 11 (1978) 2483. Unfortunately, the expression of $A_{2}$ obtained in this article is given in terms of different variables and does not appear in the same scaling form. For this reason, the results cannot be easily compared. 